\title{
Application of a Generalized SEIR Model for COVID-19 in Algeria
}

\author{
Mohamed Lounis ${ }^{1 *}$, Juarez dos Santos Azevedo ${ }^{2}$
}

${ }^{1}$ Department of Agro-veterinary Science, Faculty of Natural and Life Sciences, University of Ziane Achour, BP 3117, Road of Moudjbara, Djelfa 17000, ALGERIA ${ }^{2}$ Federal University of Bahia (UFBA), Institute of Sciences, Technology and Innovation, Centro, 42802-721, Camaçari-BA, BRAZIL

*Corresponding Author: lounisvet@gmail.com

Citation: Lounis, M. and dos Santos Azevedo, J. (2021). Application of a Generalized SEIR Model for COVID-19 in Algeria. European Journal of Sustainable Development Research, 5(1), em0150. https://doi.org/10.21601/ejosdr/9675

\section{ARTICLE INFO}

Received: 23 Aug. 2020

Accepted: 15 Nov. 2020

\begin{abstract}
The novel coronavirus disease 2019 (COVID-19) reported in Wuhan is continuing to impress the world by its fast spread and the number of affected persons attracting an unprecedented attention.

In this article, the classical SEIR model and a generalized SEIR model called SEIRDP were applied to predict the evolution of COVID-19 in Algeria for a future period of 100 days using official reported data from early April to early August, 2020. Initial evaluation showed that the two models had a net correspondence with the reported data during this period for cumulative infected cases but the number of cumulative deaths was underestimated with the classical SEIR model. Model prediction with the SEIRDP concluded that the number of cumulative infected cases will increase in the next days reaching a number of about $60 \mathrm{k}$ in middle November with a median of about 300 daily cases. Also, the number of estimated deaths will be around $2 \mathrm{k}$. These results suggest that the COVID-19 is ongoing to infect more persons which may push national authorities to carefully act in the probable leaving of containment.
\end{abstract}

Keywords: epidemic model, COVID-19, SEIR model, SEIRDP model, Algeria

\section{INTRODUCTION}

The coronavirus disease 2019 (COVID-19) detected first in Wuhan, in the province of Hubei (China) on December 2019 has provoked a real confusion in the world (Lounis, 2020). This respiratory disease caused by a novel virus called SARS-CoV2 (Severe Acute Respiratory Syndrom coronavirus 2), has afflicted more than 19.4 millions positive cases and more than $723 \mathrm{k}$ deaths around the world (Johns Hopkins University of Medicine, n.d.).

Since the first case reported on February $25^{\text {th }}$, Algeria accounts currently 34,693 cases and 1,293 deaths (Algerian health and hospital reform minister: Carte épidémiologique, n.d.).

Due to the early and the drastic implemented measures in Algeria, the situation seemed to be under control until the last April and the beginning of May (Lounis, 2020). However, the evolution of the epidemic in the last two months has shown a real increasing in the daily reported cases. This may be related to the increasing in the total number of test performed but also to a result of the alleviation of the prevention strategy and the subsequent lightening in the respect of physical distancing and protection measures (Lounis, 2020; Rouabah et al., 2020). In this way, comprehension of the epidemic curves and forecasting its evolution is very important for the evaluation of the implemented measures and in deciding to adopt the best future strategies to curb the spreading of the disease. In this way, statistics or mathematical modeling is of great importance and represents a crucial tool in understanding the epidemic characteristics and predicting its curve (Tan and Chen, 2020).

Multiple models have been achieved in Algeria to forecast the epidemic or to evaluate the preventive implemented measure with different approaches including local, regional and global studies (Balah and Djeddou, 2020; Belkacem, 2020; Ben Hassen et al., 2020; Bentout et al., 2020; Boudrioua and Boudrioua, 2020; Daw and El Bouzebdei, 2020; Hamidouche, 2020; Moussaoui and Auger, 2020; Nail et al., 2020; Rahmani, 2020; Rezki, 2020; Rouabah et al., 2020; Zhao et al., 2020). These studies used the most common SIR (Boudrioua and Boudrioua, 2020; Rezki, 2020) and SEIR (Belkacem, 2020; Bentout et al., 2020; Moussaoui and Auger, 2020; Peng et al., 2020; Rouabah et al., 2020) models or other mathematical models such as ARFIMA (Autoregressive Fractionally Integrated Moving Average Models) (Balah and Djeddou, 2020) and the Alg-COVID-19 Model (Rahmani, 2020).

In the current study, the classical model and a generalized SEIR model called SEIRDP (Susceptible, Exposed, Infected, Recovered, Death and Insusceptible (P) were used to predict the evolution of the epidemic curves in Algeria for a future period of 100 days. 


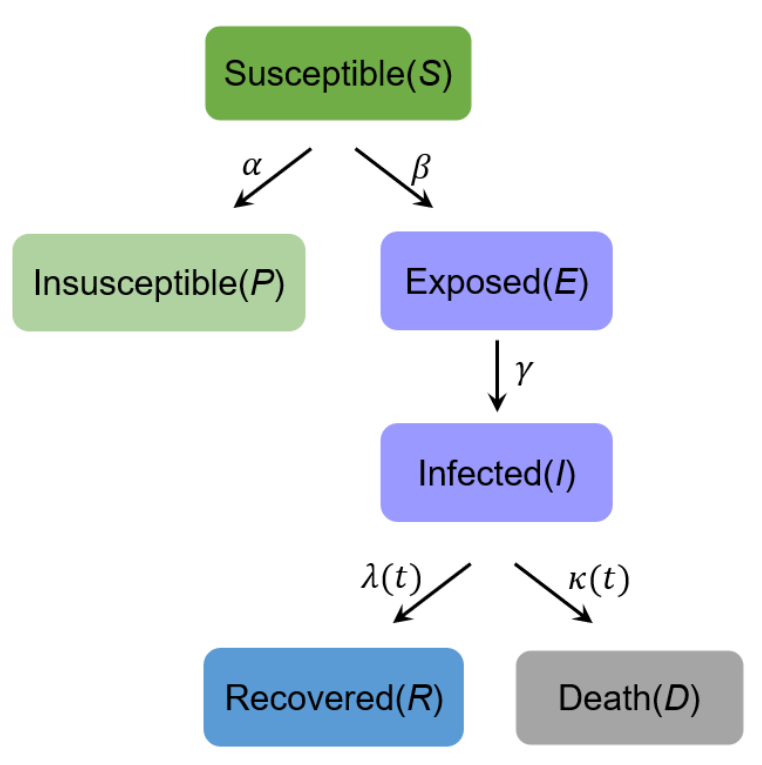

Figure 1. Scheme of the compartments of SEIRDP model

\section{Data Sources}

The daily COVID-19 new cases (confirmed with RT-PCR test) in Algeria were collected from the Ministry of Population Health and Hospital Reform official website (https://www.covid19.gov.dz/carte/) from April 1 1, 2020 to August $4^{\text {th }}, 2020$ for a period of 126 days. These data include the total positive cases, the daily positive cases, the total deaths, the new daily deaths, the total recovered, the daily recovered and at last the number of quarantined persons.

The total case in the mentioned period is of 31788 cases with an average of about 252 cases per day.

\section{METHODS}

The classical Susceptible Exposed Infectious Recovered (SEIR) model represents one of the most adopted mathematical models for characterizing and forecasting the epidemic diseases (Rouabah et al., 2020; Tan and Chen, 2020). It is widely used to study the COVID-19 curve in multiple countries in the world (Rouabah et al., 2020).

In the current study, the authors followed the adapted SEIR model developed by Tan and Chen (2020), the most reliable response to emergency public health actions which takes into account the population exposed to the virus.

More precisely, they used the generalized version of the classical SEIR called SEIRDP (Susceptible, Exposed, Infected, Recovered, Death, Insusceptible (P)) a particular type of the proposed model of Peng et al. (2020) (See Figure 1).

In the SEIRDP model, multiple key parameters of the COVID-19 epidemic like the latency time, the infection time, the protection rate and the recovery can be included. This will help to estimate the peak of the epidemic, the end-time and the total infected cases in Algeria basing on the Ministry of Health of Algeria data.

\section{SEIRDP Model Characteristics}

In the current study, the used SEIRDP model is divided to the following categories: The Susceptible population $(S)$, the Exposed population $(E)$ to the virus which is not yet infectious, the Infected population (I) confirmed in laboratory which has a potential infection, the Recovered population $(R)$, the Death number $(D)$ and the Insusceptible population $(P)$.

The total population $(N)=S+P+E+I+R+D$.

The SEIRDP model is described as follow:

$$
\begin{gathered}
\frac{\mathrm{dS}(\mathrm{t})}{d(t)}=-\beta \frac{S(t) I(t)}{N}-\alpha S(t), \\
\frac{d E(t)}{d(t)}=-\beta \frac{S(t) I(t)}{N}-\gamma E(t), \\
\frac{d I(t)}{d(t)}=-\gamma E(t)-\lambda(t) I(t)-\kappa(t) I(t), \\
\frac{d R(t)}{d(t)}=\lambda(t) I(t), \\
\frac{d D(t)}{d(t)}=\kappa(t) I(t) \\
\frac{d P(t)}{d(t)}=\alpha S(t)
\end{gathered}
$$

Where:

$\alpha$ is the protection rate including individuals exposed to both infectious and asymptomatic patients); $\beta$ represents the infection rate; $\gamma^{-1}$ is the average latency time; and $\lambda(t)$ and $\kappa(\mathrm{t})$ which are coefficients used in the time-dependent cure and mortality rate respectively.

The cure rate $\lambda(t)$ is defined according to time by:

$$
\lambda(t)=\lambda_{0} /\left[1+\exp \left(-\lambda_{1}\left(t-\lambda_{2}\right)\right)\right]
$$

and the mortality rate $\kappa(\mathrm{t})$ as:

$$
\kappa(t)=\kappa_{0} \exp \left(-\kappa_{1} t\right)
$$

These two functions are adjusted according to the cases number for $t \in\left[t_{0} ; t_{f}\right]$, with $t_{0}$ as the initial period and $t_{f}$ which represents the final period.

As described in Equation (2) and Equation (3) the rate of recovery $\lambda(t)$ grows exponentially to reach a limit value while the death rate $\kappa(t)$ tends toward zero.

Also, the least square sense method was used to adjust the $\left\{\alpha, \beta, \gamma^{-1}, \lambda(t), \kappa(t)\right\}$ parameters. Taking in consideration the starting guess $\left[\lambda_{0} ; \lambda_{1} ; \lambda_{2}\right]=[0: 1 ; 0: 1 ; 0: 1]$ and $\left[\kappa_{0} ; \kappa_{1}\right]=[0: 5 ; 0: 3]$, these functions are plotted as shown in Figure 2.

A nonlinear least-squares solver was used to compute the parameters $\left\{\alpha, \beta, \gamma^{-1}, \lambda_{0}, \lambda_{1}, \lambda_{2}, \kappa_{0}(t), \kappa_{1}(t)\right\}$ (Cheynet, 2020).

These parameters can be replaced in the model (1) and the time-histories of the different states $\{S(t) ; P(t) ; E(t)$; $I(t) ; R(t) ; D(t)\}$ can be calculated. Here, the standard fourthorder Runge-Kutta process was used (Burden et al., 2015) and the set of equations is written as a single ODE as:

$$
\frac{d Y}{d t}=A \cdot Y+F
$$

$\mathrm{Y}=[S(t) E(t) I(t) R(t) D(t) P(t)]^{\mathrm{T}}$ 


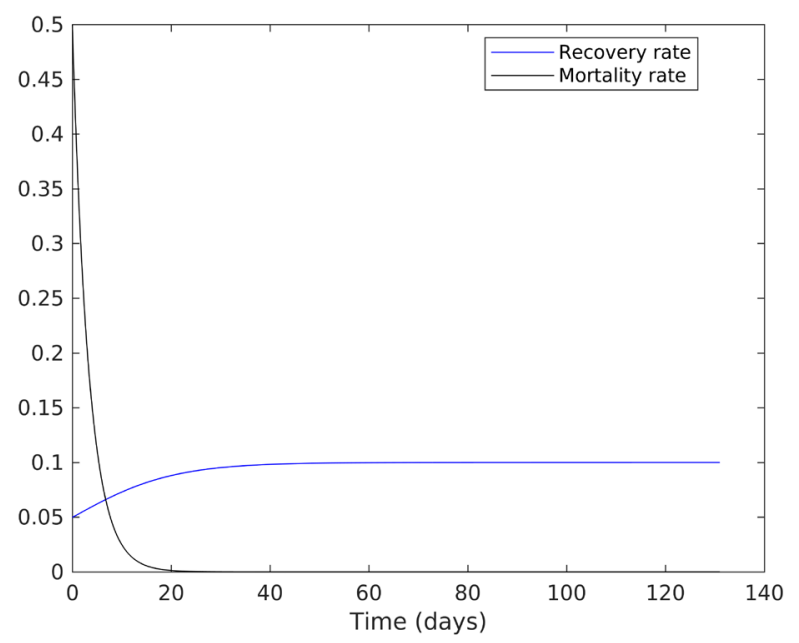

Figure 2. Plot of recovery and mortality rates



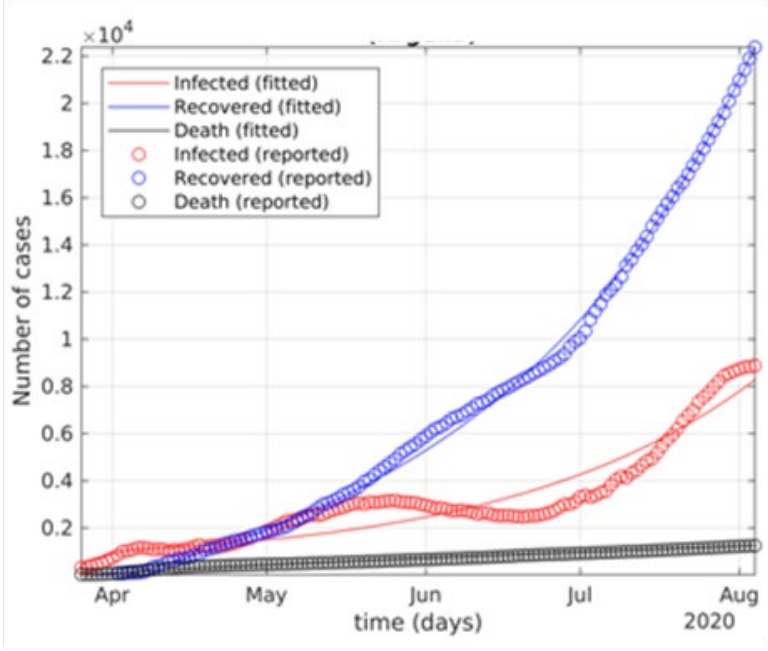

(a)

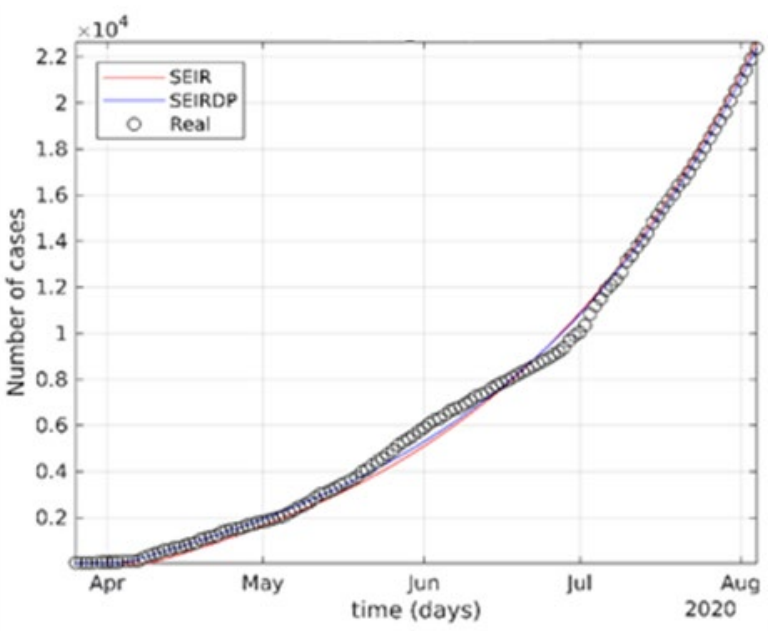

(c)

$$
F=S(t) \cdot I(t) \cdot\left[-\frac{\beta}{N} \quad \frac{\beta}{N} \quad 0 \quad 00 \quad 0 \quad 0 .\right]_{T}
$$

\section{RESULTS AND DISCUSSION}

\section{Prediction of the Evolution of COVID-19 in Algeria}

Here the authors present the time series obtained by the SEIRDP model. The prediction of the Algerian situation according to this model shows the trends given in Figure 3.

The prediction of this model from early April, 2020 to early August, 2020 showed that this model has a good correspondence with the data reported in the same period by the ministry of health (Algerian health and hospital reform minister: Carte épidémiologique, n.d.) in term of total, infected, recovery and death cases (Figure 3a). This indicates that the parameters of the SEIRDP model could be useful to predict the evolution of the COVID-19 epidemic in the future.

When compared with the traditional SEIR, results showed that the prediction of the SEIR model was in correspondence with the prediction of SEIRDP and the reported cumulative cases (see Figure $3 \mathbf{b}$ ) and recovered cases (see Figure $3 \mathbf{b}$ ) by

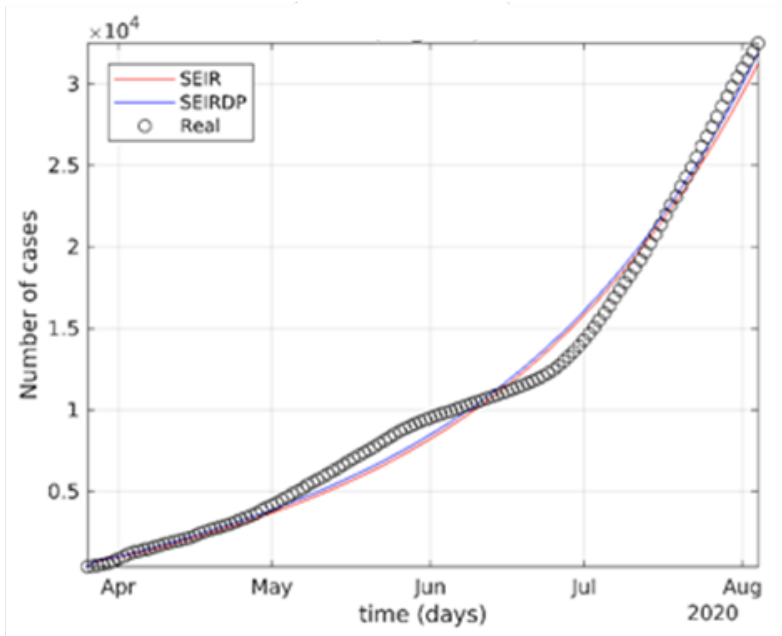

(b)

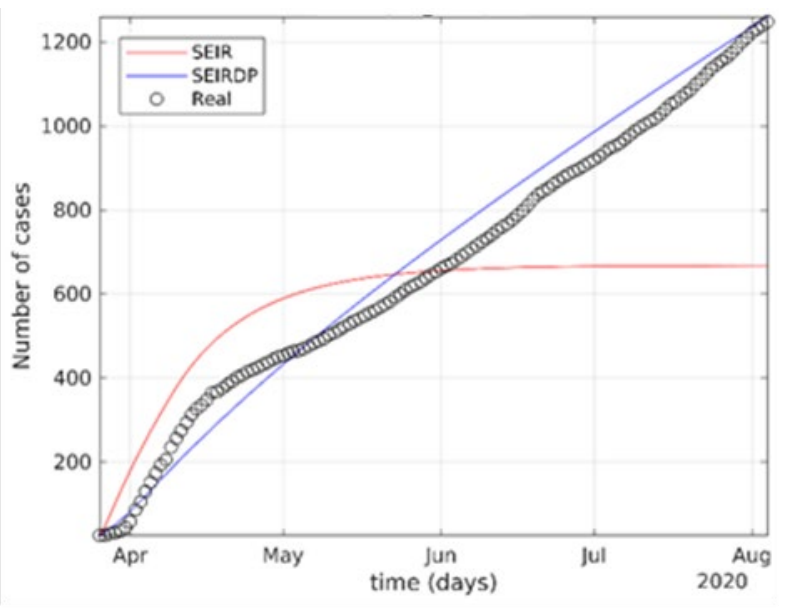

(d)

Figure 3. (a) Modeling of the differential SEIRDP model from April 1 to August 3 (c) Modeling of the differential SEIR model from April 1 to August 3 for cumulative positive cases (b), recovered (c), cumulative deaths (d). 


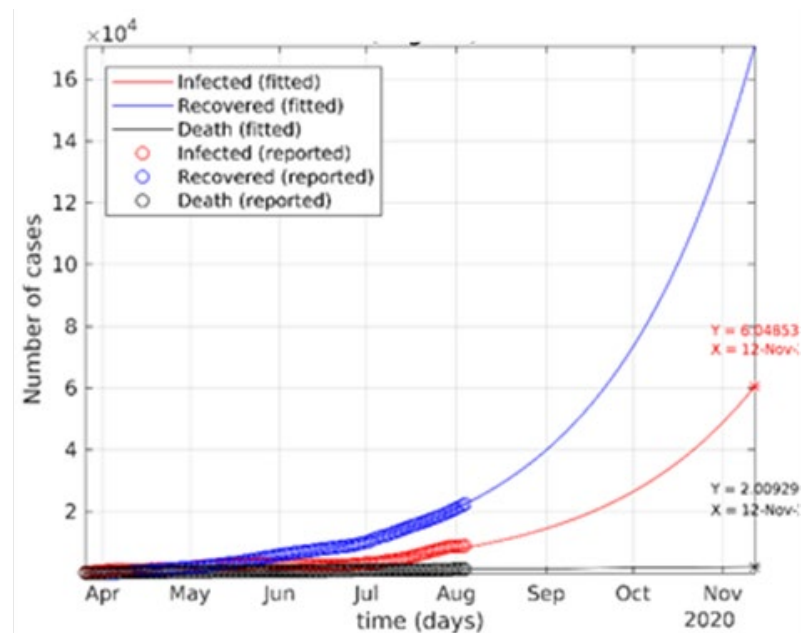

(a)

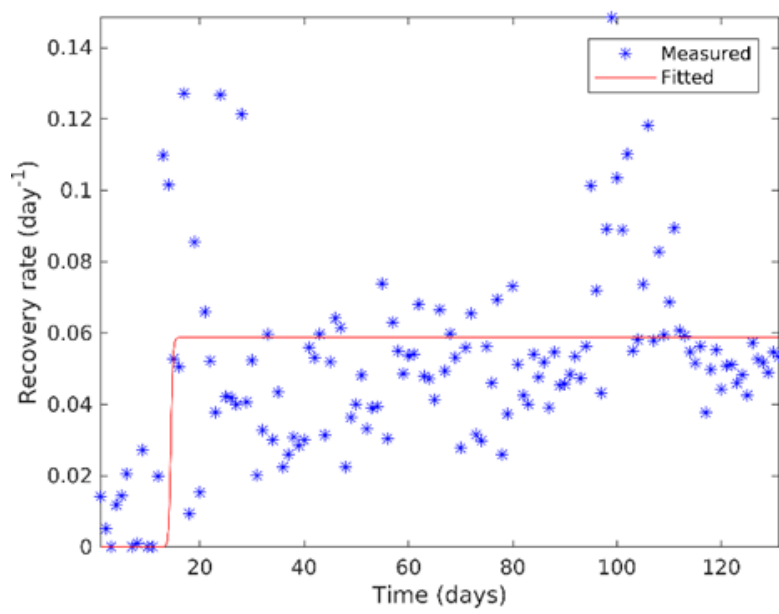

(c)

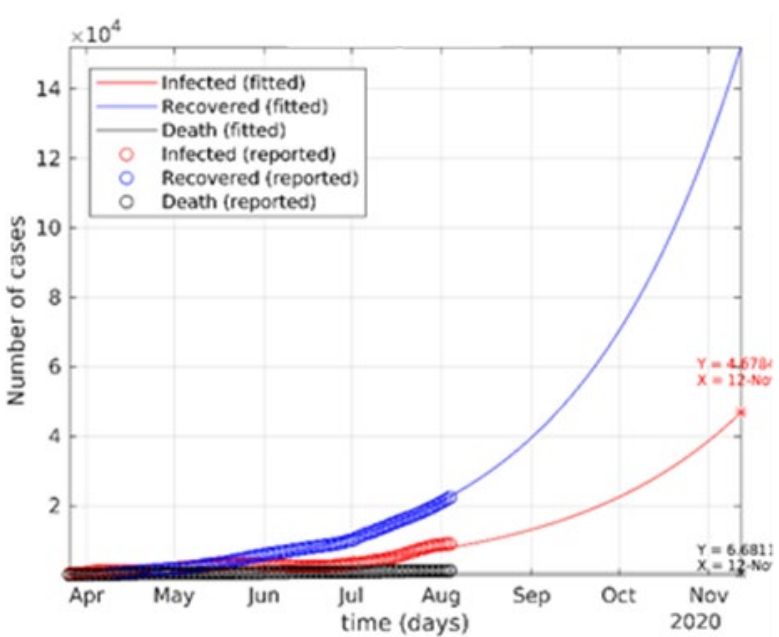

(b)

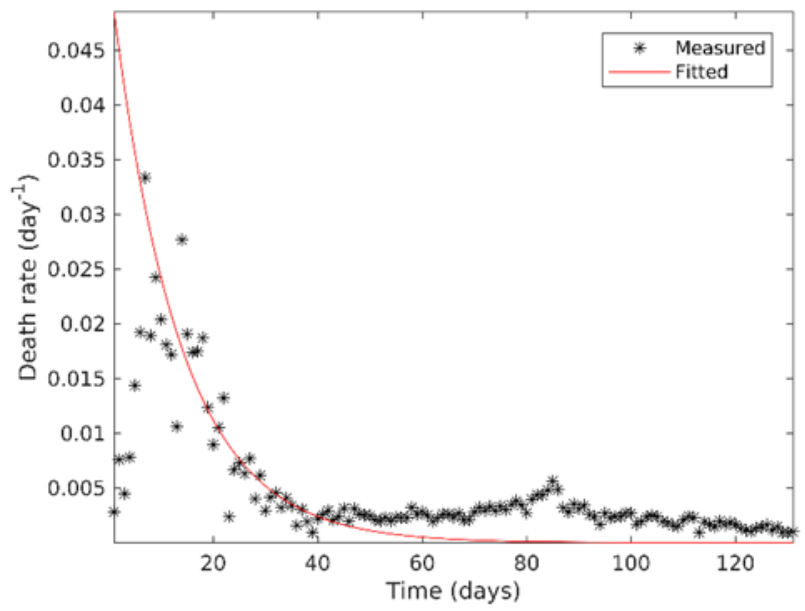

(d)

Figure 4. (a) Predictions of the differential SEIRDP model for Algeria from early April to mid-November, 2020. (b) Predictions of the differential SEIR model for Algeria from early April to mid-November, 2020. (c) The recovery rates and (d) deceased rates of April to early-August, 2020

the ministry of health, while the number of predicted deaths was underestimated (see Figure 3d).

The SEIRDP model was later used to predict the evolution of the pandemic for from April to November, 2020. Results showed that the number of cases in Algeria still increasing to attempt a number of about $60 \mathrm{k}$ positive cases and about $2 \mathrm{k}$ deaths in $12^{\text {th }}$ November, 2020 (see Figure 4a. Prediction of the classical SEIR model however, estimated the number of cumulative cases at about 46k (see Figure 4b. In Figures 4c4d, we have the recovery and death rates as a function of time respectively. In this case, results between the computed and observed rates were compared.

In Figure $4 \mathrm{~b}$ we can observe a constant behavior while in Figure 4c we have an exponential decrease in mortality rates as time progresses.

These results corroborate with the plots of Figure 2.

At last, it is to note that, the current model is using the official data by the Ministry of healthy based only of RT-PCR testing. The number of performed tests was very low in the first days (about 60 tests/day). This number has currently attempt 2500 tests/day but remain low for screening the total cases which could affect the total number of cases in the model.

\section{CONCLUSION}

In this study, the authors used a classical and a generalized SEIR models to analyze the epidemic curve COVID-19 and its evolution in Algeria.

Based on data reports of the Ministry of public health and hospital reform from early April to early August, it was first shown that the two models, the classical SEIR model and the SEIRDP model had a good correspondence with the daily reported data in term of cumulative infected cases and recovered while the classical SEIR model underestimated the cumulative deaths. The SEIRDP model prediction for the next 100 days showed that the number of cases will increase to reach a number of $60 \mathrm{k}$ in middle November while the predicted number of deceased persons will be around $2 \mathrm{k}$ cases. The number of cumulative cases with the classical SEIR model will reach a number of about $46 \mathrm{k}$ in the middle November. These results showed that the number of cases is still increasing in Algeria despite the implemented measures which may be taken in consideration public authority in the next steps of fighting this disease. 


\section{REFERENCES}

Algerian health and hospital reform minister: Carte épidémiologique. Available at: https://www.COVID19.gov. dz/carte/ (Accessed: 8 August 2020).

Balah, B. and Djeddou, M. (2020). Forecasting COVID-19 new cases in Algeria using Autoregressive fractionally integrated moving average Models (ARFIMA). medRxiv preprint. https://doi.org/10.1101/2020.05.03.20089615

Belkacem, S. (2020, 19 Jul). COVID-19 data analysis and forecasting: Algeria and the world. arXiv: 2007.09755v1 [stat.AP].

Ben Hassen, H., Elaoud, A., Ben Salah, N. and Masmoudi, A. (2020). A SIR-Poisson Model for COVID-19: Evolution and Transmission Inference in the Maghreb Central Regions. Arab. J. Sci. Engin, 46, 93-102. https://doi.org/10.1007/ s13369-020-04792-0

Bentout, S., Chekroun, A. and Kuniya, T. (2020). Parameter estimation and prediction for coronavirus disease outbreak 2019 (COVID-19) in Algeria. AIMS Public Health, 7(2), 306318. https://doi.org/10.3934/publichealth.2020026

Boudrioua, M. S. and Boudrioua, A. (2020). Predicting the COVID-19 epidemic in Algeria using the SIR model. medRxiv preprint. https://doi.org/10.1101/2020.04.25. 20079467

Burden, R. L., Faires, J. D. and Burden, A. M. (2015). Numerical Analysis. Cengage Learning.

Cheynet, E. (2020, Apr). Generalized SEIR Epidemic Model (fitting and computation). https://www.github.com/ ECheynet/SEIR

Daw, M. A. and El Bouzebdei, A. H. (2020). Modelling the epidemic spread of COVID-19 virus infection in Northern African countries. Travel Med. Inf. Dis., 35, 101671. https://doi.org/10.1016/j.tmaid.2020.101671

Hamidouche, M. (2020). COVID-19 Outbreak in Algeria: A Model to Predict Cumulative Cases. Journal of Contemporary Studies in Epidemiology and Public Health, 1(1), ep20004. https://doi.org/10.30935/jconseph/8451
Johns Hopkins University of Medicine, Coronavirus resource center: https://coronavirus.jhu.edu/map.html (Accessed: 8 August 2020).

Lounis, M. (2020). A Descriptive Study of the Current Situation of COVID-19 in Algeria. Electronic Journal of General Medicine, 17(6), em253. https://doi.org/10.29333/ ejgm/8287

Moussaoui, A. and Auger, P. (2020). Prediction of confinement effects on the number of COVID-19 outbreak in Algeria. Math. Model. Nat. Phenom., 15, 37. https://doi.org/ 10.1051/mmnp/2020028

Nail, B., Rabehi, A., Bekhiti, B. and Arbaoui, T. (2020). A new design of an adaptive model of infectious diseasesbased on artificial intelligence approach: monitoring andforecasting of COVID-19 epidemic cases. medRxiv preprint. https://doi.org/10.1101/2020.04.23.20077677

Peng, L., Yang, W., Zhang, D., Zhuge, C. and Hong, L. (2020). Epidemic analysis of COVID-19 in China by dynamical modeling. arXiv preprint: 2002.06563. https://doi.org/ 10.1101/2020.02.16.20023465

Rahmani, S. E. A. (2020). Spatial distribution of COVID-19, a modelingapproach: case of Algeria. Research Square. https://doi.org/10.21203/rs.3.rs-40447/v1

Rezki, M. (2020). Estimating the total size of coronavirus epidemic in Algeria via different approaches. medRxiv preprint. https://doi.org/10.1101/2020.07.29.20164509

Rouabah, M. T., Tounsi, A. and Belaloui, N. E. (2020). A mathematical epidemic model using genetic fitting algorithm with cross-validation and application to early dynamics of COVID-19 in Algeria. arXiv: 2005.13516v3 [qbio.PE] 24 Jun 2020.

Tan, S. X. D. and Chen, L. (2020). Real-time differential epidemic analysis and prediction for COVID-19 pandemic. arXiv preprint:2004.06888.

Zhao, Z., Li, X., Liu, F., Zhu, G., Ma, C. and Wang, L. (2020). Prediction of the COVID-19 spread in African countries and implications for prevention and controls: A case study in South Africa, Egypt, Algeria, Nigeria, Senegal and Kenya. Sci. Total Env, 729, 138959. https://doi.org/10.1016/ j.scitotenv.2020.138959 